\title{
Unilateral and Bilateral Aplasia of the Anterior Cerebral Artery in the Presence of Their Vascular Sources
}

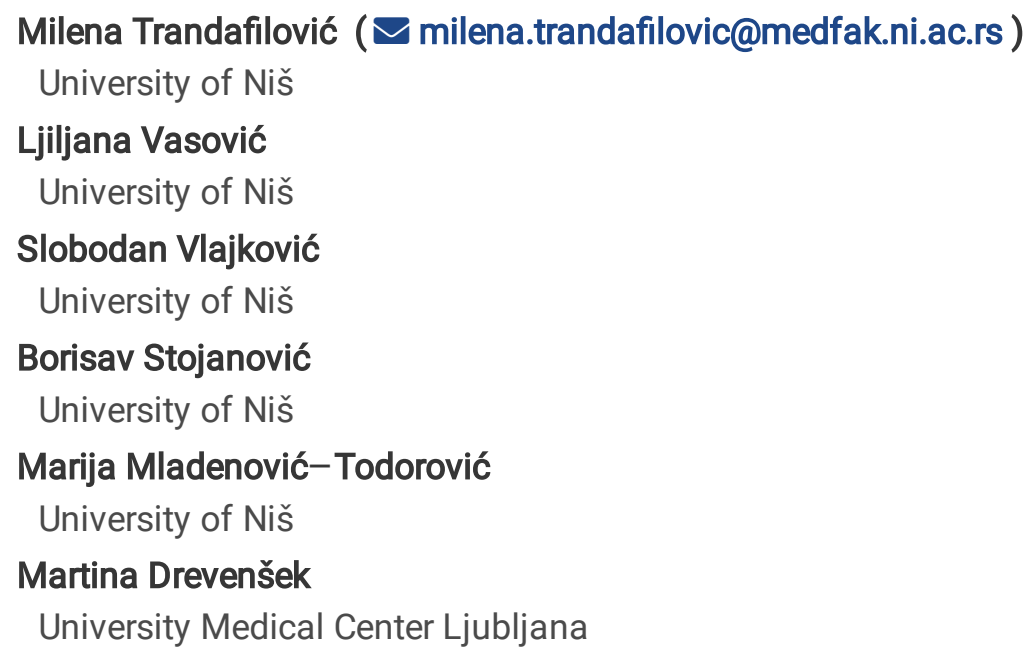

\section{Research Article}

Keywords: Adult human cadaver, Brain base, Anterior cerebral artery, Aplasia, Associated abnormalities

Posted Date: March 7th, 2022

DOI: https://doi.org/10.21203/rs.3.rs-1413223/v1

License: (9) (1) This work is licensed under a Creative Commons Attribution 4.0 International License. Read Full License 


\section{Abstract \\ Purpose}

In continuation of our previous studies of vascular components of the cerebral arterial circle, our goal was to investigate the cases of aplasia of the anterior cerebral artery (ACA).

\section{Methods}

The macro- and microdissection of the target human brain arteries was applied under the magnifying glass. Each case was photographed and diagramatically represented in the workbook. The length and the outer diameter of the corresponding arteries on the photos were measured using a computer software program.

\section{Results}

There were $2 / 388$ cases of ACA aplasia- unilaterally in one case $(0.25 \%)$, and bilaterally in the other $(0.25 \%)$. These two cases belonged to a male and a female adult, autopsied due to the homicide and myocardial infarction, aged 68 and 80 , respectively. Except for the variations of the posterior communicating artery on one or both sides, and several arteries of the vertebrobasilar system, the instance of cerebral pathology was not recorded in either of these cases. We compared the recent cases with similar literature cases obtained from a number of retrospective studies and single reports.

\section{Conclusion}

Summarizing available literature cases of unilateral ACA aplasia, and taking into account that the presented cases of unilateral and bilateral ACA aplasia were not accompanied with cerebral pathology, we can nevertheless to underline the appearance of an aneurysm of the opposite ACA in more than a quarter of the studied cases, more typically on the right side and in male gender. The recent case of bilateral ACA aplasia which has been the second case discovered so far, represents a true rarity.

\section{Introduction}

When the embryo is approximately $40 \mathrm{~mm}$ long (ovulation age - 52), as cited by Burbank and Morris [5], the cerebral arterial circle (CAC) could be recognized and the stems of all cerebral arteries attain an adult configuration.

The paired anterior cerebral arteries (ACAs) with their pre-communicating parts together with the anterior communicating artery (ACoA) between them, as well as with their vascular sources, i.e. the cerebral part (C4) of both of the internal carotid arteries (ICAs) participate in the form of the so-called anterior segment of the CAC [53].

There are data that the pre-communicating part of the ACA had the medium length (caliber) of 14.7 (2.2) $\mathrm{mm}$ on the right side and 13.8 (2.4) $\mathrm{mm}$ on the left side in adult cadaveric specimens [21], and the medium diameter of $2.1 \mathrm{~mm}$ on both sides in patients aged up to 40 , and $1.9 \mathrm{~mm}$ on the right and $2.0 \mathrm{~mm}$ on the left side in patients being older than 40 years [6].

The trunk of the ACA is divided in the pre-communicating (A1) and the post-communicating (A2) parts in relation to the ACoA. It distributes central and cortical branches that supply many subcortical structures, as well as the gyri and lobules of the frontal, lymbic and parietal lobes on the inferior and medial surfaces of the hemisphere. It distributes anteromedial central branches in the A1 part; the proximal medial striate arteries from the previous group of branches supply the anterior hypothalamus, the septum pellucidum, the anterior cerebral commissure, the fornix and the striatum, while the preoptic, 
supraoptic and the anterior perforating arteries, also from the previous group of branches, supply the corresponding nuclei of diencephalon [9].

However, there were descriptions of many morphologic variations of the ACA, such as the origin of both of the ACAs from one ICA [5, 23, 40], or A1 aplasia [4, 10, 15, 27, 29, 37], or hypoplasia [1, 8, 17, 22, 27, 28, 44, 49, 51, 52], or an unusual course $[23,36,42]$, or distibution of variable branches [26, 38, 51], or a fenestration $[11,13,20,38,49,53]$, or a nonunion with the opposite ACA [38, 51], or forming of the so-called azygos pericallosal artery $[11,38,47,50]$, or tripling of the A2 parts [1, 11, $47,50]$. Many authors found an association between pathologic disorders and ACA variations $[19,24,29,39,46,47,54]$.

The aim of this paper was to investigate the incidence of unilateral or bilateral aplasia of the ACA, as well as potentially associated vascular variants and/or pathologic disorders in these cases.

\section{Material And Methods}

The research work was performed on the brains of 388 human cadavers of both genders and of different ages (from neonate to 95), routinely autopsied due to different reasons or pathology.

The research of 332 brains was performed during the author's graduate and postgraduate studies under the supervision of two professors of Anatomy; the approvals were obtained from the Council of Graduate Studies of Faculty of Medicine and the Head of the Institute of Forensic Medicine, while an additional approval was obtained from the Research Ethics Committee (No. 01-9068-4) of our Faculty of Medicine. The research of 55 adult cerebral arteries was performed in the Institute of Forensic Medicine in Niš during the previous graduate studies of two co-authors (BS and MM) under the supervision of the same professors of Anatomy in the period of 2011 to 2015; the approvals were obtained also from the Council of Graduate Studies of Faculty of Medicine and the Director of the Institute of Forensic Medicine.

Each single brain base with blood vessels of all the cases was recorded on the photo film and schematically presented in the workbook. The morphological status of the ACA was inspected macroscopically and by magnifying glass; the outer diameters (ODs) of the cerebral arteries were calculated using the digital images, via the ImageJ processing program (http://rsb.info.nih.gov/ij/index.html).

\section{Results}

\section{The findings of retrospective study}

We found one case (1/388 cadavers or $0.25 \%)$ or $1 / 776$ ACAs with unilateral aplasia, and one case $(1 / 388$ or $0.25 \%)$ with bilateral aplasia of the ACA. We incorporated our findings to the sheet of the similar literature data in Table 1.

Table 1 Incidence of aplasia of the pre-communicating (A1) part of the anterior cerebral artery (ACA) in the literature ${ }^{\mathrm{a}}$ and recent studies 


\begin{tabular}{|c|c|c|c|c|c|c|c|}
\hline \multirow{2}{*}{$\begin{array}{l}\mathrm{N}^{0} \\
\text { of } \\
\text { stu- } \\
\text { dies }\end{array}$} & \multirow{2}{*}{$\begin{array}{l}\text { Coun- } \\
\text { try (author) }\end{array}$} & \multirow{2}{*}{$\begin{array}{l}\mathrm{N}^{\circ} \text { of cases of the both genders } \\
\text { (with or without evidenced } \\
\text { pathology) }\end{array}$} & \multirow{2}{*}{$\begin{array}{l}\text { Age } \\
\text { of patients } \\
\text { or cadavers }\end{array}$} & \multirow{2}{*}{$\begin{array}{l}\text { Diag-nostic } \\
\text { proce-dure }\end{array}$} & \multicolumn{3}{|c|}{ Incidence of aplasia } \\
\hline & & & & & Left & Right & Bilateral \\
\hline 1 & $\begin{array}{l}\text { Japan } \\
{[54]}\end{array}$ & $\begin{array}{l}9 \\
\text { (patients with diagnosed A1 } \\
\text { aneurysms) }\end{array}$ & $43-77$ & $\begin{array}{l}\text { MIP MRA / } \\
\text { Ope-rative fin- } \\
\text { ding }\end{array}$ & $(11.1 \%)^{\mathrm{b}}$ & & \\
\hline 2 & $\begin{array}{l}\text { Pakistan } \\
{[46]}\end{array}$ & $\begin{array}{l}54 \\
\text { (patients with hemorrhagic } \\
\text { stroke) }\end{array}$ & $20-70$ & CTA & $\begin{array}{l}1 \\
(1.8 \%)\end{array}$ & $\begin{array}{l}1 \\
(1.8 \%)\end{array}$ & \\
\hline 3 & $\begin{array}{l}\text { Japan } \\
\text { [34] }\end{array}$ & $\begin{array}{l}55 \text { patients } \\
\text { (instrumentation of the posterior } \\
\text { cervical spine) }\end{array}$ & wd & 3D CTA & $(3.6 \%)$ & & \\
\hline 4 & $\begin{array}{l}\text { India } \\
\text { [43] }\end{array}$ & $\begin{array}{l}70 \\
\text { (patients) }\end{array}$ & wd & $\begin{array}{l}\text { 3D TOF } \\
\text { MRA / } \\
\text { 3D MIP MRA } \\
/ \\
\text { VRT }\end{array}$ & $\begin{array}{l}3 \\
(4.3 \%)^{\mathrm{b}}\end{array}$ & & \\
\hline 5 & $\begin{array}{l}\text { USA } \\
\text { [22] }\end{array}$ & $\begin{array}{l}104 \\
\text { (angiograms of patients without } \\
\text { AVM or intracranial hemorrhage) }\end{array}$ & $3-84$ & MRA / MRI & 6 & 4 & \\
\hline 6 & $\begin{array}{l}\text { India } \\
{[16]}\end{array}$ & $\begin{array}{l}112 \\
\text { (cadavers) }\end{array}$ & wd & $\begin{array}{l}\text { Anat. } \\
\text { dissec-tion }\end{array}$ & $\begin{array}{l}1 \\
(0.9 \%)\end{array}$ & $\begin{array}{l}1 \\
(0.9 \%)\end{array}$ & \\
\hline 7 & $\begin{array}{l}\text { Sudan } \\
\text { [2] }\end{array}$ & $\begin{array}{l}143 \\
\text { (patients without cerebrovascular } \\
\text { accidents) }\end{array}$ & wd & MRA & $\begin{array}{l}3 \\
(2.1 \%)\end{array}$ & $\begin{array}{l}3 \\
(2.1 \%)\end{array}$ & \\
\hline 8 & $\begin{array}{l}\text { Germany } \\
\text { [39] }\end{array}$ & $\begin{array}{l}155 \\
\text { (patients with aneurysms } \\
\text { of ACA) }\end{array}$ & wd & & $\begin{array}{l}11 \\
(7.1 \%)^{b}\end{array}$ & & \\
\hline 9 & $\begin{array}{l}\text { Turkey } \\
{[1]}\end{array}$ & $\begin{array}{l}75 \\
\text { (patients with aneurysms } \\
\text { of } A C O A \text { ) }\end{array}$ & $3-89$ & DSA & $\begin{array}{l}6 \\
(20 \%)\end{array}$ & 9 & \\
\hline & & $\begin{array}{l}107 \\
\text { (control group without vascular } \\
\text { pathology) }\end{array}$ & & & $(0.9 \%)$ & $(0.9 \%)$ & \\
\hline 10 & $\begin{array}{l}\text { Nether- } \\
\text { lands }\end{array}$ & $\begin{array}{l}208 \\
\text { (patients) }\end{array}$ & $29-79$ & MRA & 11 & & \\
\hline
\end{tabular}




\begin{tabular}{|c|c|c|c|c|c|c|c|}
\hline 11 & [28] & $\begin{array}{l}\text { (patients without cerebral } \\
\text { pathology) }\end{array}$ & $(x)$ & TCD / MRA & \multicolumn{2}{|l|}{$\begin{array}{l}(6.0 \%) \\
(8 M+6 F)\end{array}$} & \\
\hline 12 & $\begin{array}{l}\text { Poland } \\
\text { [27] }\end{array}$ & $\begin{array}{l}411 \\
\text { (patients without vascular } \\
\text { pathology) }\end{array}$ & $\begin{array}{l}47.6 \\
(x)\end{array}$ & CTA & $(4.6 \%)$ & & \\
\hline 13 & $\begin{array}{l}\text { USA } \\
{[14]}\end{array}$ & $\begin{array}{l}414 \\
\text { (cadavers) }\end{array}$ & wd & Auto-psy & & & 2 \\
\hline 14 & $\begin{array}{l}\text { Taiwan } \\
\text { [6] }\end{array}$ & $\begin{array}{l}111 \\
\text { (patients without cerebral } \\
\text { pathology) }\end{array}$ & $<40$ & $\begin{array}{l}\text { MRI / } \\
\text { MRA }\end{array}$ & $(3.60 \%)$ & & \\
\hline & & $\begin{array}{l}396 \\
\text { (patients without cerebral } \\
\text { pathology) }\end{array}$ & $>40$ & & $(11.62 \%)$ & & \\
\hline 15 & $\begin{array}{l}\text { Japan } \\
{[47]}\end{array}$ & $\begin{array}{l}891 \\
\text { (patients suspected to } \\
\text { cerebrovascular disease) }\end{array}$ & $0-92$ & MRA & $\begin{array}{l}19 \\
(5.6 \%)^{b}\end{array}$ & 31 & \\
\hline 16 & $\begin{array}{l}\text { Serbia } \\
{[19]}\end{array}$ & 1000 angiograms & $\begin{array}{l}61.7 \\
(x)\end{array}$ & MRA & $\begin{array}{l}12 \\
(1.2 \%)\end{array}$ & $\begin{array}{l}17 \\
(1.7 \%)\end{array}$ & \\
\hline 17 & $\begin{array}{l}\text { Our } \\
\text { study }\end{array}$ & 377 & $0-95$ & $\begin{array}{l}\text { Auto- } \\
\text { psy }\end{array}$ & $\begin{array}{l}1 \\
(0.26 \%)\end{array}$ & & $\begin{array}{l}1 \\
(0.26 \%)\end{array}$ \\
\hline
\end{tabular}

aLiterature studies are aligned according to the number of investigated cases.

${ }^{\mathrm{b}}$ The incidence is calculated according to presented number of cases in corresponding articles.

Maximum intensity projection magnetic resonance angiography (MIP MRA); computed tomography arteriography (CTA); without data (wd); three-dimensional time-of-flight magnetic resonance angiography (3D TOF MRA); volume rendering technique (VRT); arteriovenous malformation (AVM); magnetic resonance image (MRI); digital subtraction angiography (DSA); transcranial Doppler (TCD); male (M); female (F); post-communicating part (A2) of the anterior cerebral artery; anterior communicating artery (ACOA).

\section{The description of the analyzed two cases}

Unilateral A1 aplasia was found on the left ACA in an 80-year-old female autopsied due to myocardial infarction. There was a cerebral arterial semicircle (CAS) on the brain base formed by both of the C4 parts, and the right ACA (in the so-called anterior segment of the CAS), and the paired posterior communicating arteries (PCoAs) and the pre-communicating parts (P1s) of the posterior cerebral arteries (PCAs) in the so-called posterior segment of the CAS. The right ACA with the OD of $3.18 \mathrm{~mm}$ originated from the ipsilateral ICA $(O D=5.27 \mathrm{~mm})$, which was relatively larger than the left ICA $(O D=4.41 \mathrm{~mm})$. The right ACA coursed anteromedially to the longitudinal fissure along the length of $13.42 \mathrm{~mm}$, when it bifurcated in the cistern of lamina terminalis in the left $(\mathrm{OD}=2.13 \mathrm{~mm})$ and the right $(\mathrm{OD}=1.89 \mathrm{~mm})$ pericallosal arteries (PAs). There was an oval fenestration $2.47 \mathrm{~mm}$ long in the trunk of the right ACA before its bifurcation. The right PA had a short trunk that trifurcated; one of these branches reached the opposite side and coursed along medial surface of the left hemisphere 
together with the left PA. Additionally, there was bilateral hypoplasia $(0.60 / 0.64 \mathrm{~mm})$ of the PCoA, and the right double superior cerebellar arteries arose from the ipsilateral pre-communicating part (P1) of the PCA (Fig. 1). We found no aneurysm of the right ACA nor of other cerebral arteries in this case.

Bilateral A1 aplasia and the ensuing absence of the ACoA were found in a 68-year-old male that was autopsied due to the homicide. Except for the hypoplastic caliber of the left PCOA $(0.89 \mathrm{~mm})$ and an ectasia of the basilar artery $(O D=4.81 \mathrm{~mm})$, the ODs of the ICAs and the arteries of the posterior segment of the CAS did not significantly differ from each other (Fig. 2). We found no aneurysms of the other cerebral arteries in this case. However, there were skeletal and visceral abnormalities (pelvic deformity, a shorter right lower limb, small sized kidneys and basocellular carcinoma of the face).

We introduced the general features of each single (recent and literature) case of unilateral and bilateral ACA aplasia in Table 2. 
Table 2

Literature $^{a}$ and recent single cases of aplasia of the pre-communicating (A1) part of anterior cerebral artery (ACA)

\begin{tabular}{|c|c|c|c|c|c|c|c|c|c|}
\hline \multirow{2}{*}{$\begin{array}{l}\text { No } \\
\text { of } \\
\text { ca- } \\
\text { ses }\end{array}$} & \multirow{2}{*}{$\begin{array}{l}\text { Country } \\
\text { [author] }\end{array}$} & \multirow{2}{*}{$\begin{array}{l}\text { Age of } \\
\text { patient/ } \\
\text { gender }\end{array}$} & \multirow{2}{*}{$\begin{array}{l}\text { Initial } \\
\text { symptoms } \\
\text { or reason } \\
\text { of } \\
\text { research }\end{array}$} & \multirow{2}{*}{$\begin{array}{l}\text { Diagnostic } \\
\text { procedure }\end{array}$} & \multicolumn{3}{|c|}{ A1 aplasia } & \multirow{2}{*}{$\begin{array}{l}\text { Associated } \\
\text { variations }^{\mathrm{b}}\end{array}$} & \multirow{2}{*}{$\begin{array}{l}\text { Associated } \\
\text { pathology }\end{array}$} \\
\hline & & & & & Left & Right & $\begin{array}{l}\text { Bi- } \\
\text { la- } \\
\text { te- } \\
\text { ral }\end{array}$ & & \\
\hline 1 & $\begin{array}{l}\text { USA } \\
{[5]}\end{array}$ & $2 / F$ & Seizures & $\begin{array}{l}\text { MRI / 3D } \\
\text { TOF MRA }\end{array}$ & + & & & $\begin{array}{l}\text { Left ACA } \\
\text { originated } \\
\text { from the right } \\
\text { ICA } \\
\text { close to the } \\
\text { ophthalmic } \\
\text { artery origin }\end{array}$ & $\begin{array}{l}\text { Congenital } \\
\text { disorders: } \\
\text { Chiari II } \\
\text { malformation / } \\
\text { spina bifida / } \\
\text { hydrocephalus. } \\
\text { Acquired } \\
\text { disorder: left- } \\
\text { sided cerebral } \\
\text { infarct. }\end{array}$ \\
\hline 2 & $\begin{array}{l}\text { USA } \\
\text { [22] }\end{array}$ & $28 / \mathrm{M}$ & Headache & $\begin{array}{l}\text { Axial T1- } \\
\text { weighted } \\
\text { spin-echo } \\
\text { MRI / 3D } \\
\text { TOF MRA }\end{array}$ & + & & & $\begin{array}{l}\text { Hypoplastic } \\
\text { left ICA }\end{array}$ & \\
\hline 3 & $\begin{array}{l}\text { Japan } \\
\text { [23] }\end{array}$ & $48 / \mathrm{M}$ & Headache & $\begin{array}{l}\text { CT / CTA / } \\
\text { Operative } \\
\text { finding }\end{array}$ & + & & & $\begin{array}{l}\text { Left ACA } \\
\text { originated } \\
\text { from the right } \\
\text { ICA close to } \\
\text { the } \\
\text { ophtalmic } \\
\text { artery origin. } \\
\text { Infraoptic } \\
\text { course, and } \\
\text { irregular wall } \\
\text { of the right } \\
\text { ACA }\end{array}$ & $\begin{array}{l}\text { Aneurysm of } \\
\text { the right A1. } \\
\text { SAH. }\end{array}$ \\
\hline 4 & $\begin{array}{l}\text { India } \\
\text { [20] }\end{array}$ & $50 / F$ & $\begin{array}{l}\text { Headache } \\
\text { / vomiting }\end{array}$ & $\begin{array}{l}\text { CT / } \\
\text { Cerebral } \\
\text { four-vessel } \\
\text { angiography } \\
\text { / Operative } \\
\text { finding }\end{array}$ & + & & & $\begin{array}{l}\text { Fenestration } \\
\text { of the right } \\
\text { A1 }\end{array}$ & $\begin{array}{l}\text { Aneurysm of } \\
\text { the right A1 (at } \\
\text { proximal end } \\
\text { of } \\
\text { fenestration). } \\
\text { SAH. }\end{array}$ \\
\hline
\end{tabular}

aLiterature cases are aligned according to the age of individuals; some cases originated from the retrospective studies, in which A1 aplasia was marked in single angiograms [22, 28, 43, 47, 54].

${ }^{\mathrm{b}}$ Associated vascular variations and pathology are noted according to their description in original articles.

'Fisher [14] sketched two cases of bilateral partial aplasia of the ACAs in A1 parts.

${ }^{\mathrm{d}}$ Recent cases are presented in two gray rows.

Female (F); magnetic resonance image (MRI); three dimensional time-of-flight magnetic resonance angiography (3D TOF MRA); internal carotid artery (ICA); male (M); computed tomography (CT); computed tomography angiography (CTA); cervical (C); subarachnoid hemorrhage (SAH); post-communicating part (A2) of the anterior cerebral artery; posterior communicating artery $(\mathrm{PCOA})$; anterior communicating artery $(\mathrm{ACOA})$; maximum intensity projection magnetic resonance angiography (MIP MRA); multidetector computed tomography angiography (MD CTA); transcranial Doppler (TCD); digital subtraction angiography (DSA); two (three) dimensional transcranial color-coded sonography (2D (3D) TCCS)); vertebrobasilar system (VBS); middle cerebral artery (MCA). 


\begin{tabular}{|c|c|c|c|c|c|c|c|c|c|}
\hline \multirow{2}{*}{$\begin{array}{l}\text { No } \\
\text { of } \\
\text { ca- } \\
\text { ses }\end{array}$} & \multirow{2}{*}{$\begin{array}{l}\text { Country } \\
\text { [author] }\end{array}$} & \multirow{2}{*}{$\begin{array}{l}\text { Age of } \\
\text { patient/ } \\
\text { gender }\end{array}$} & \multirow{2}{*}{$\begin{array}{l}\text { Initial } \\
\text { symptoms } \\
\text { or reason } \\
\text { of } \\
\text { research }\end{array}$} & \multirow{2}{*}{$\begin{array}{l}\text { Diagnostic } \\
\text { procedure }\end{array}$} & \multicolumn{3}{|c|}{ A1 aplasia } & \multirow{2}{*}{$\begin{array}{l}\text { Associated } \\
\text { variations }^{\mathrm{b}}\end{array}$} & \multirow{2}{*}{$\begin{array}{l}\text { Associated } \\
\text { pathologyb }\end{array}$} \\
\hline & & & & & Left & Right & $\begin{array}{l}\text { Bi- } \\
\text { la- } \\
\text { te- } \\
\text { ral }\end{array}$ & & \\
\hline 5 & $\begin{array}{l}\text { Denmark } \\
\text { [42] }\end{array}$ & $55 / F$ & SAH & $\begin{array}{l}\text { Carotid / } \\
\text { vertebral } \\
\text { angiography } \\
\text { / Operative } \\
\text { finding }\end{array}$ & + & & & $\begin{array}{l}\text { Preoptic } \\
\text { origin of the } \\
\text { right ACA } \\
\text { from } \\
\text { ipsilateral } \\
\text { ICA. } \\
\text { Position of } \\
\text { the right } \\
\text { A1part } \\
\text { between two } \\
\text { optic nerves }\end{array}$ & $\begin{array}{l}\text { Aneurysm of } \\
\text { the ACoA. }\end{array}$ \\
\hline 6 & $\begin{array}{l}\text { Japan } \\
\text { [54] }\end{array}$ & $56 / \mathrm{M}$ & SAH & MRI / MRA & + & & & & $\begin{array}{l}\text { Aneurysm of } \\
\text { the right } \mathrm{A} 1 \\
\text { (middle part) }\end{array}$ \\
\hline 7 & $\begin{array}{l}\text { India } \\
\text { [35] }\end{array}$ & $60 / \mathrm{M}$ & Cadaver & Dissection & + & & & $\begin{array}{l}\text { Left ICA of a } \\
\text { small caliber. } \\
\text { Aplasia of } \\
\text { the left PCoA. }\end{array}$ & \\
\hline 8 & $\begin{array}{l}\text { UK } \\
\text { [12] }\end{array}$ & $63 / \mathrm{F}$ & $\begin{array}{l}\text { Left } \\
\text { hemipa- } \\
\text { resis / } \\
\text { right leg } \\
\text { weakness }\end{array}$ & 3D CTA & + & & & & $\begin{array}{l}\text { Aneurysm } \\
\text { of the ACoA. } \\
\text { Bilateral } \\
\text { anterior } \\
\text { cerebral } \\
\text { infarctions. }\end{array}$ \\
\hline 9 & $\begin{array}{l}\text { Japan } \\
\text { [47] }\end{array}$ & $67 / M$ & $\begin{array}{l}\text { Cerebral } \\
\text { infarction }\end{array}$ & MIP MRA & + & & & $\begin{array}{l}\text { Small right } \\
\text { A2 }\end{array}$ & \\
\hline 10 & $\begin{array}{l}\text { USA } \\
\text { [3] }\end{array}$ & $76 / \mathrm{M}$ & $\begin{array}{l}\text { Numbness } \\
\text { / } \\
\text { weakness } \\
\text { of lower } \\
\text { extremi- } \\
\text { ties }\end{array}$ & $\begin{array}{l}\text { Cerebral } \\
\text { angiography }\end{array}$ & + & & & & $\begin{array}{l}\text { Right ICA } \\
\text { stenosis }\end{array}$ \\
\hline
\end{tabular}

a Literature cases are aligned according to the age of individuals; some cases originated from the retrospective studies, in which $A 1$ aplasia was marked in single angiograms $[22,28,43,47,54]$.

${ }^{\mathrm{b}}$ Associated vascular variations and pathology are noted according to their description in original articles.

'Fisher [14] sketched two cases of bilateral partial aplasia of the ACAs in A1 parts.

${ }^{\mathrm{d}}$ Recent cases are presented in two gray rows.

Female (F); magnetic resonance image (MRI); three dimensional time-of-flight magnetic resonance angiography (3D TOF MRA); internal carotid artery (ICA); male (M); computed tomography (CT); computed tomography angiography (CTA); cervical (C); subarachnoid hemorrhage (SAH); post-communicating part (A2) of the anterior cerebral artery; posterior communicating artery $(\mathrm{PCOA})$; anterior communicating artery $(\mathrm{ACOA})$; maximum intensity projection magnetic resonance angiography (MIP MRA); multidetector computed tomography angiography (MD CTA); transcranial Doppler (TCD); digital subtraction angiography (DSA); two (three) dimensional transcranial color-coded sonography (2D (3D) TCCS)); vertebrobasilar system (VBS); middle cerebral artery (MCA). 


\begin{tabular}{|c|c|c|c|c|c|c|c|c|c|}
\hline \multirow{2}{*}{$\begin{array}{l}\text { No } \\
\text { of } \\
\text { ca- } \\
\text { ses }\end{array}$} & \multirow{2}{*}{$\begin{array}{l}\text { Country } \\
\text { [author] }\end{array}$} & \multirow{2}{*}{$\begin{array}{l}\text { Age of } \\
\text { patient/ } \\
\text { gender }\end{array}$} & \multirow{2}{*}{$\begin{array}{l}\text { Initial } \\
\text { symptoms } \\
\text { or reason } \\
\text { of } \\
\text { research }\end{array}$} & \multirow{2}{*}{$\begin{array}{l}\text { Diagnostic } \\
\text { procedure }\end{array}$} & \multicolumn{3}{|c|}{ A1 aplasia } & \multirow{2}{*}{$\begin{array}{l}\text { Associated } \\
\text { variations }\end{array}$} & \multirow{2}{*}{$\begin{array}{l}\text { Associatec } \\
\text { pathology }\end{array}$} \\
\hline & & & & & Left & Right & $\begin{array}{l}\text { Bi- } \\
\text { la- } \\
\text { te- } \\
\text { ral }\end{array}$ & & \\
\hline 11 & $\begin{array}{l}\text { India } \\
{[40]}\end{array}$ & - & Cadaver & Dissection & + & & & $\begin{array}{l}\text { The both } \\
\text { ACA } \\
\text { originated } \\
\text { from the right } \\
\text { ICA }\end{array}$ & \\
\hline 12 & $\begin{array}{l}\text { India } \\
\text { [43] }\end{array}$ & - & Image & MRA & + & & & $\begin{array}{l}\text { The both } \\
\text { ACA } \\
\text { originated } \\
\text { from the right } \\
\text { ICA }\end{array}$ & \\
\hline 13 & $\begin{array}{l}\text { Turkey } \\
\text { [38] }\end{array}$ & - & Image & 3D CTA & + & & & & \\
\hline
\end{tabular}

aLiterature cases are aligned according to the age of individuals; some cases originated from the retrospective studies, in which A1 aplasia was marked in single angiograms $[22,28,43,47,54]$.

${ }^{\mathrm{b}}$ Associated vascular variations and pathology are noted according to their description in original articles.

'Fisher [14] sketched two cases of bilateral partial aplasia of the ACAs in A1 parts.

${ }^{\mathrm{d}}$ Recent cases are presented in two gray rows.

Female (F); magnetic resonance image (MRI); three dimensional time-of-flight magnetic resonance angiography (3D TOF MRA); internal carotid artery (ICA); male (M); computed tomography (CT); computed tomography angiography (CTA); cervical (C); subarachnoid hemorrhage (SAH); post-communicating part (A2) of the anterior cerebral artery; posterior communicating artery $(\mathrm{PCOA})$; anterior communicating artery $(\mathrm{ACOA})$; maximum intensity projection magnetic resonance angiography (MIP MRA); multidetector computed tomography angiography (MD CTA); transcranial Doppler (TCD); digital subtraction angiography (DSA); two (three) dimensional transcranial color-coded sonography (2D (3D) TCCS)); vertebrobasilar system (VBS); middle cerebral artery (MCA). 


\begin{tabular}{|c|c|c|c|c|c|c|c|c|c|}
\hline \multirow{2}{*}{$\begin{array}{l}\text { No } \\
\text { of } \\
\text { ca- } \\
\text { ses }\end{array}$} & \multirow{2}{*}{$\begin{array}{l}\text { Country } \\
\text { [author] }\end{array}$} & \multirow{2}{*}{$\begin{array}{l}\text { Age of } \\
\text { patient/ } \\
\text { gender }\end{array}$} & \multirow{2}{*}{$\begin{array}{l}\text { Initial } \\
\text { symptoms } \\
\text { or reason } \\
\text { of } \\
\text { research }\end{array}$} & \multirow{2}{*}{$\begin{array}{l}\text { Diagnostic } \\
\text { procedure }\end{array}$} & \multicolumn{3}{|c|}{ A1 aplasia } & \multirow{2}{*}{$\begin{array}{l}\text { Associated } \\
\text { variations }^{b}\end{array}$} & \multirow{2}{*}{$\begin{array}{l}\text { Associated } \\
\text { pathology }\end{array}$} \\
\hline & & & & & Left & Right & $\begin{array}{l}\text { Bi- } \\
\text { la- } \\
\text { te- } \\
\text { ral }\end{array}$ & & \\
\hline 14 & $\begin{array}{l}\text { Australia } \\
{[11]}\end{array}$ & - & Image & MDCTA & + & & & & \\
\hline 15 & $\begin{array}{l}\text { France } \\
{[36]}\end{array}$ & - & Image & $\begin{array}{l}\text { 1.5-Tesla } \\
\text { MRI }\end{array}$ & + & & & $\begin{array}{l}\text { Infraoptic } \\
\text { course of the } \\
\text { right A1. } \\
\text { Plexular } \\
\text { ACoA }\end{array}$ & \\
\hline 16 & $\begin{array}{l}\text { Korea } \\
{[28]}\end{array}$ & - & Image & MRA / TCD & + & & & & \\
\hline 17 & $\begin{array}{l}\text { Germany } \\
\text { [24] }\end{array}$ & - & Image & $\begin{array}{l}\text { DSA / 2D } \\
\text { (3D) TCCS }\end{array}$ & + & & & & $\begin{array}{l}\text { Aneurysm } \\
\text { of the ACoA }\end{array}$ \\
\hline 18 & $\begin{array}{l}\text { USA } \\
\text { [29] }\end{array}$ & - & Image & DSA / CTA & + & & & & $\begin{array}{l}\text { Aneurysm } \\
\text { of the right } \\
\text { ACA }\end{array}$ \\
\hline 19 & $\begin{array}{l}\text { Taiwan } \\
\text { [6] }\end{array}$ & - & Image & MRA & + & & & & \\
\hline 20 & $\begin{array}{l}\text { Serbia } \\
\text { (recent } \\
\text { case) }\end{array}$ & $80 / F$ & $\begin{array}{l}\text { Cadaver } \\
\text { (myo- } \\
\text { cardial } \\
\text { infarction) }\end{array}$ & $\begin{array}{l}\text { Forensic } \\
\text { autopsy }\end{array}$ & + & & & $\begin{array}{l}\text { Fenestration } \\
\text { of the right } \\
\text { ACA. } \\
\text { Hypoplasia } \\
\text { of the both } \\
\text { PCoAs; } \\
\text { initial partial } \\
\text { duplication } \\
\text { of the left } \\
\text { PCoA. }\end{array}$ & $\begin{array}{l}\text { Atheromatous } \\
\text { plaques in } \\
\text { ICAs, the right } \\
\text { ACA and } \\
\text { branches of } \\
\text { the VBS } \\
\text { system. }\end{array}$ \\
\hline
\end{tabular}

aLiterature cases are aligned according to the age of individuals; some cases originated from the retrospective studies, in which $A 1$ aplasia was marked in single angiograms $[22,28,43,47,54]$.

${ }^{\mathrm{b}}$ Associated vascular variations and pathology are noted according to their description in original articles.

'Fisher [14] sketched two cases of bilateral partial aplasia of the ACAs in A1 parts.

${ }^{\mathrm{d}}$ Recent cases are presented in two gray rows.

Female (F); magnetic resonance image (MRI); three dimensional time-of-flight magnetic resonance angiography (3D TOF MRA); internal carotid artery (ICA); male (M); computed tomography (CT); computed tomography angiography (CTA); cervical (C); subarachnoid hemorrhage (SAH); post-communicating part (A2) of the anterior cerebral artery; posterior communicating artery $(\mathrm{PCOA})$; anterior communicating artery $(\mathrm{ACOA})$; maximum intensity projection magnetic resonance angiography (MIP MRA); multidetector computed tomography angiography (MD CTA); transcranial Doppler (TCD); digital subtraction angiography (DSA); two (three) dimensional transcranial color-coded sonography (2D (3D) TCCS)); vertebrobasilar system (VBS); middle cerebral artery (MCA). 


\begin{tabular}{|c|c|c|c|c|c|c|c|c|c|}
\hline \multirow{2}{*}{$\begin{array}{l}\text { No } \\
\text { of } \\
\text { ca- } \\
\text { ses }\end{array}$} & \multirow{2}{*}{$\begin{array}{l}\text { Country } \\
\text { [author] }\end{array}$} & \multirow{2}{*}{$\begin{array}{l}\text { Age of } \\
\text { patient/ } \\
\text { gender }\end{array}$} & \multirow{2}{*}{$\begin{array}{l}\text { Initial } \\
\text { symptoms } \\
\text { or reason } \\
\text { of } \\
\text { research }\end{array}$} & \multirow{2}{*}{$\begin{array}{l}\text { Diagnostic } \\
\text { procedure }\end{array}$} & \multicolumn{3}{|c|}{ A1 aplasia } & \multirow{2}{*}{$\begin{array}{l}\text { Associated } \\
\text { variations }^{\text {b }}\end{array}$} & \multirow{2}{*}{$\begin{array}{l}\text { Associated } \\
\text { pathologyb }^{\text {bath }}\end{array}$} \\
\hline & & & & & Left & Right & $\begin{array}{l}\text { Bi- } \\
\text { la- } \\
\text { te- } \\
\text { ral }\end{array}$ & & \\
\hline 21 & $\begin{array}{l}\text { UK } \\
\text { [31] }\end{array}$ & Neonatus & Image & MIP MRA & & + & & $\begin{array}{l}\text { Bilateral fetal } \\
\text { origin of the } \\
\text { PCA }\end{array}$ & \\
\hline 22 & $\begin{array}{l}\text { Germany } \\
\text { [15] }\end{array}$ & $25 / \mathrm{M}$ & Accident & $\begin{array}{l}\text { Carotid } \\
\text { angiography }\end{array}$ & & + & & Right PPHA & \\
\hline 23 & $\begin{array}{l}\text { India } \\
\text { [26] }\end{array}$ & $35 / \mathrm{M}$ & Headache & $\begin{array}{l}\text { Cerebral } \\
\text { angiography } \\
\text { / Operative } \\
\text { finding }\end{array}$ & & + & & $\begin{array}{l}\text { Left } \\
\text { callosomargi- } \\
\text { nal artery } \\
\text { originated } \\
\text { from the left } \\
\text { A1 }\end{array}$ & $\begin{array}{l}\text { Aneurysm } \\
\text { of the left A1 }\end{array}$ \\
\hline 24 & $\begin{array}{l}\text { UK } \\
{[10]}\end{array}$ & $47 / M$ & $\begin{array}{l}\text { Left arm } \\
\text { weakness } \\
\text { / } \\
\text { dysarthria }\end{array}$ & 3D CTA & & + & & $\begin{array}{l}\text { Hypoplastic } \\
\text { right ICA; } \\
\text { right CCA } \\
\text { bifurcated at } \\
\text { the level of } \\
\text { the C VI } \\
\text { vertebra. }\end{array}$ & \\
\hline 25 & $\begin{array}{l}\text { USA } \\
{[45]}\end{array}$ & $54 / \mathrm{M}$ & $\begin{array}{l}\text { Aphasia / } \\
\text { dysarthria } \\
\text { / right } \\
\text { hemipa- } \\
\text { resis }\end{array}$ & MRI / MRA & & + & & Left PPTA & \\
\hline 26 & $\begin{array}{l}\text { India } \\
\text { [41] }\end{array}$ & $58 / \mathrm{M}$ & $\begin{array}{l}\text { Giddiness } \\
\text { / headache }\end{array}$ & $\begin{array}{l}\text { Cerebral } \\
\text { four-vessel } \\
\text { angiography } \\
\text { / Operative } \\
\text { finding }\end{array}$ & & + & & & $\begin{array}{l}\text { Aneurysm } \\
\text { of the left A1 }\end{array}$ \\
\hline
\end{tabular}

a Literature cases are aligned according to the age of individuals; some cases originated from the retrospective studies, in which A1 aplasia was marked in single angiograms $[22,28,43,47,54]$.

${ }^{\mathrm{b}}$ Associated vascular variations and pathology are noted according to their description in original articles.

'Fisher [14] sketched two cases of bilateral partial aplasia of the ACAs in A1 parts.

${ }^{\mathrm{d}}$ Recent cases are presented in two gray rows.

Female (F); magnetic resonance image (MRI); three dimensional time-of-flight magnetic resonance angiography (3D TOF MRA); internal carotid artery (ICA); male (M); computed tomography (CT); computed tomography angiography (CTA); cervical (C); subarachnoid hemorrhage (SAH); post-communicating part (A2) of the anterior cerebral artery; posterior communicating artery $(\mathrm{PCOA})$; anterior communicating artery $(\mathrm{ACOA})$; maximum intensity projection magnetic resonance angiography (MIP MRA); multidetector computed tomography angiography (MD CTA); transcranial Doppler (TCD); digital subtraction angiography (DSA); two (three) dimensional transcranial color-coded sonography (2D (3D) TCCS)); vertebrobasilar system (VBS); middle cerebral artery (MCA). 


\begin{tabular}{|c|c|c|c|c|c|c|c|c|c|}
\hline \multirow{2}{*}{$\begin{array}{l}\text { No } \\
\text { of } \\
\text { ca- } \\
\text { ses }\end{array}$} & \multirow{2}{*}{$\begin{array}{l}\text { Country } \\
\text { [author] }\end{array}$} & \multirow{2}{*}{$\begin{array}{l}\text { Age of } \\
\text { patient/ } \\
\text { gender }\end{array}$} & \multirow{2}{*}{$\begin{array}{l}\text { Initial } \\
\text { symptoms } \\
\text { or reason } \\
\text { of } \\
\text { research }\end{array}$} & \multirow{2}{*}{$\begin{array}{l}\text { Diagnostic } \\
\text { procedure }\end{array}$} & \multicolumn{3}{|c|}{ A1 aplasia } & \multirow{2}{*}{$\begin{array}{l}\text { Associated } \\
\text { variations }^{\mathrm{b}}\end{array}$} & \multirow{2}{*}{$\begin{array}{l}\text { Associated } \\
\text { pathology }\end{array}$} \\
\hline & & & & & Left & Right & $\begin{array}{l}\text { Bi- } \\
\text { la- } \\
\text { te- } \\
\text { ral }\end{array}$ & & \\
\hline 27 & $\begin{array}{l}\text { Croatia } \\
{[4]}\end{array}$ & $61 / \mathrm{M}$ & $\begin{array}{l}\text { Mild } \\
\text { speech } \\
\text { disorder / } \\
\text { left supra- } \\
\text { nuclear } \\
\text { palsy }\end{array}$ & $\begin{array}{l}\text { Color } \\
\text { Doppler / } \\
\text { MRA / CTA }\end{array}$ & & + & & $\begin{array}{l}\text { Left VA } \\
\text { aplasia / } \\
\text { Right VA } \\
\text { hypoplasia / } \\
\text { Left PPIA } \\
\text { (type I) }\end{array}$ & $\begin{array}{l}\text { Right cerebral } \\
\text { infarction / } \\
\text { stenoses of the } \\
\text { both CCAs and } \\
\text { ECAs / } \\
\text { fusiform } \\
\text { aneurysm of } \\
\text { the right SA }\end{array}$ \\
\hline 28 & $\begin{array}{l}\text { Korea } \\
\text { [30] }\end{array}$ & $62 / \mathrm{M}$ & $\begin{array}{l}\text { Loss of } \\
\text { conscious- } \\
\text { ness }\end{array}$ & $\begin{array}{l}\text { 3D TOF } \\
\text { MRA }\end{array}$ & & + & & $\begin{array}{l}\text { Aplasia of } \\
\text { the left PCoA }\end{array}$ & \\
\hline 29 & $\begin{array}{l}\text { India } \\
\text { [25] }\end{array}$ & $64 / M$ & $\begin{array}{l}\text { Headache } \\
\text { / vomiting }\end{array}$ & $\begin{array}{l}\text { CT / Carotid } \\
\text { angiography }\end{array}$ & & + & & & $\begin{array}{l}\text { Bilateral } \\
\text { infarctions } \\
\text { of the frontal } \\
\text { lobe }\end{array}$ \\
\hline 30 & $\begin{array}{l}\text { India } \\
\text { [37] }\end{array}$ & $64 / M$ & $\begin{array}{l}\text { Weaknes } \\
\text { in right } \\
\text { upper and } \\
\text { lower } \\
\text { limbs }\end{array}$ & $\begin{array}{l}\text { 3D TOF } \\
\text { MRA }\end{array}$ & & + & & $\begin{array}{l}\text { Left VA as a } \\
\text { branch of the } \\
\text { CCA } \\
\text { trifurcation } \\
\text { penetrated C } \\
\text { I transverse } \\
\text { foramen. }\end{array}$ & \\
\hline 31 & $\begin{array}{l}\text { Japan } \\
{[47]}\end{array}$ & $82 / F$ & $\begin{array}{l}\text { Lacunar } \\
\text { infarction }\end{array}$ & MIP MRA & & + & & & $\begin{array}{l}\text { Aneurysm } \\
\text { of the left A1- } \\
\text { ACoA }\end{array}$ \\
\hline 32 & $\begin{array}{l}\text { Germany } \\
{[48]}\end{array}$ & - & Image & CTA & & + & & $\begin{array}{l}\text { Aplasia of } \\
\text { the right } \\
\text { PCoA }\end{array}$ & \\
\hline 33 & & - & Image & & & + & & $\begin{array}{l}\text { Hypoplasia } \\
\text { of the both } \\
\text { PCoAs }\end{array}$ & \\
\hline
\end{tabular}

aLiterature cases are aligned according to the age of individuals; some cases originated from the retrospective studies, in which $A 1$ aplasia was marked in single angiograms $[22,28,43,47,54]$.

${ }^{\mathrm{b}}$ Associated vascular variations and pathology are noted according to their description in original articles.

'Fisher [14] sketched two cases of bilateral partial aplasia of the ACAs in A1 parts.

${ }^{\mathrm{d}}$ Recent cases are presented in two gray rows.

Female (F); magnetic resonance image (MRI); three dimensional time-of-flight magnetic resonance angiography (3D TOF MRA); internal carotid artery (ICA); male (M); computed tomography (CT); computed tomography angiography (CTA); cervical (C); subarachnoid hemorrhage (SAH); post-communicating part (A2) of the anterior cerebral artery; posterior communicating artery $(\mathrm{PCOA})$; anterior communicating artery $(\mathrm{ACOA})$; maximum intensity projection magnetic resonance angiography (MIP MRA); multidetector computed tomography angiography (MD CTA); transcranial Doppler (TCD); digital subtraction angiography (DSA); two (three) dimensional transcranial color-coded sonography (2D (3D) TCCS)); vertebrobasilar system (VBS); middle cerebral artery (MCA). 


\begin{tabular}{|c|c|c|c|c|c|c|c|c|c|}
\hline \multirow{2}{*}{$\begin{array}{l}\text { No } \\
\text { of } \\
\text { ca- } \\
\text { ses }\end{array}$} & \multirow{2}{*}{$\begin{array}{l}\text { Country } \\
\text { [author] }\end{array}$} & \multirow{2}{*}{$\begin{array}{l}\text { Age of } \\
\text { patient/ } \\
\text { gender }\end{array}$} & \multirow{2}{*}{$\begin{array}{l}\text { Initial } \\
\text { symptoms } \\
\text { or reason } \\
\text { of } \\
\text { research }\end{array}$} & \multirow{2}{*}{$\begin{array}{l}\text { Diagnostic } \\
\text { procedure }\end{array}$} & \multicolumn{3}{|c|}{ A1 aplasia } & \multirow{2}{*}{$\begin{array}{l}\text { Associated } \\
\text { variations }^{\text {b }}\end{array}$} & \multirow{2}{*}{$\begin{array}{l}\text { Associated } \\
\text { pathologyb }\end{array}$} \\
\hline & & & & & Left & Right & $\begin{array}{l}\text { Bi- } \\
\text { la- } \\
\text { te- } \\
\text { ral }\end{array}$ & & \\
\hline 34 & Netherlands & - & Image & $\begin{array}{l}\text { 3D TOF } \\
\text { MRA }\end{array}$ & & + & & & \\
\hline
\end{tabular}

aLiterature cases are aligned according to the age of individuals; some cases originated from the retrospective studies, in which A1 aplasia was marked in single angiograms $[22,28,43,47,54]$.

${ }^{\mathrm{b}}$ Associated vascular variations and pathology are noted according to their description in original articles.

'Fisher [14] sketched two cases of bilateral partial aplasia of the ACAs in A1 parts.

${ }^{\mathrm{d}}$ Recent cases are presented in two gray rows.

Female (F); magnetic resonance image (MRI); three dimensional time-of-flight magnetic resonance angiography (3D TOF MRA); internal carotid artery (ICA); male (M); computed tomography (CT); computed tomography angiography (CTA); cervical (C); subarachnoid hemorrhage (SAH); post-communicating part (A2) of the anterior cerebral artery; posterior communicating artery $(\mathrm{PCOA})$; anterior communicating artery $(\mathrm{ACOA})$; maximum intensity projection magnetic resonance angiography (MIP MRA); multidetector computed tomography angiography (MD CTA); transcranial Doppler (TCD); digital subtraction angiography (DSA); two (three) dimensional transcranial color-coded sonography (2D (3D) TCCS)); vertebrobasilar system (VBS); middle cerebral artery (MCA). 


\begin{tabular}{|c|c|c|c|c|c|c|c|c|c|}
\hline \multirow{2}{*}{$\begin{array}{l}\text { No } \\
\text { of } \\
\text { ca- } \\
\text { ses }\end{array}$} & \multirow{2}{*}{$\begin{array}{l}\text { Country } \\
\text { [author] }\end{array}$} & \multirow{2}{*}{$\begin{array}{l}\text { Age of } \\
\text { patient/ } \\
\text { gender }\end{array}$} & \multirow{2}{*}{$\begin{array}{l}\text { Initial } \\
\text { symptoms } \\
\text { or reason } \\
\text { of } \\
\text { research }\end{array}$} & \multirow{2}{*}{$\begin{array}{l}\text { Diagnostic } \\
\text { procedure }\end{array}$} & \multicolumn{3}{|c|}{ A1 aplasia } & \multirow{2}{*}{$\begin{array}{l}\text { Associated } \\
\text { variations }^{b}\end{array}$} & \multirow{2}{*}{$\begin{array}{l}\text { Associated } \\
\text { pathologyb }\end{array}$} \\
\hline & & & & & Left & Right & $\begin{array}{l}\text { Bi- } \\
\text { la- } \\
\text { te- } \\
\text { ral }\end{array}$ & & \\
\hline 38 & $\begin{array}{l}\text { Serbia } \\
{[19]}\end{array}$ & - & Image & MRA & & + & & & \\
\hline 39 & $\begin{array}{l}\text { Korea } \\
\text { [7] }\end{array}$ & $64 / \mathrm{M}$ & Vertigo & $\begin{array}{l}\text { MRI / } \\
\text { Cerebral } \\
\text { angiography }\end{array}$ & & & + & \multicolumn{2}{|l|}{$\begin{array}{l}\text { Leptomenin- } \\
\text { geal } \\
\text { collaterals } \\
\text { from the both } \\
\text { MCAs }\end{array}$} \\
\hline $40^{c}$ & \multirow{2}{*}{$\begin{array}{l}\text { USA } \\
\text { [14] }\end{array}$} & - & Cadaver & \multirow[t]{2}{*}{ Autopsies } & & & + & & \\
\hline $41^{\mathrm{c}}$ & & - & Cadaver & & & & + & & \\
\hline 42 & $\begin{array}{l}\text { Serbia } \\
\text { (recent } \\
\text { case) }^{d}\end{array}$ & $68 / \mathrm{M}$ & $\begin{array}{l}\text { Cadaver } \\
\text { (homicide) }\end{array}$ & $\begin{array}{l}\text { Forensic } \\
\text { autopsy }\end{array}$ & & & + & \multicolumn{2}{|l|}{$\begin{array}{l}\text { Left PCoA } \\
\text { hypoplasia. } \\
\text { BA ectasia. }\end{array}$} \\
\hline \multicolumn{10}{|c|}{$\begin{array}{l}\text { a Literature cases are aligned according to the age of individuals; some cases originated from the retrospective studies, } \\
\text { in which A1 aplasia was marked in single angiograms }[22,28,43,47,54] \text {. }\end{array}$} \\
\hline \multicolumn{10}{|c|}{ bAssociated vascular variations and pathology are noted according to their description in original articles. } \\
\hline \multicolumn{10}{|c|}{ 'Fisher [14] sketched two cases of bilateral partial aplasia of the ACAs in A1 parts. } \\
\hline \multicolumn{10}{|c|}{${ }^{\mathrm{d}}$ Recent cases are presented in two gray rows. } \\
\hline \multicolumn{10}{|c|}{$\begin{array}{l}\text { Female (F); magnetic resonance image (MRI); three dimensional time-of-flight magnetic resonance angiography (3D } \\
\text { TOF MRA); internal carotid artery (ICA); male (M); computed tomography (CT); computed tomography angiography } \\
\text { (CTA); cervical (C); subarachnoid hemorrhage (SAH); post-communicating part (A2) of the anterior cerebral artery; } \\
\text { posterior communicating artery (PCoA); anterior communicating artery (ACoA); maximum intensity projection magnetic } \\
\text { resonance angiography (MIP MRA); multidetector computed tomography angiography (MD CTA); transcranial Doppler } \\
\text { (TCD); digital subtraction angiography (DSA); two (three) dimensional transcranial color-coded sonography (2D (3D) } \\
\text { TCCS)); vertebrobasilar system (VBS); middle cerebral artery (MCA). }\end{array}$} \\
\hline
\end{tabular}

\section{ACA aplasia vs. ACA aneurysm}

The cases of ACA aneurysms in the groups with the unilateral A1 aplasia, and the cases of unilateral aplasia in the groups of ACA aneurysms are selected from the previous two tables and displayed in Table 3. 
Table 3

Relationship of incidence of aplasia of the pre-communicating (A1) part of the anterior cerebral artery (ACA) in groups with ACA aneurysms and vice versa

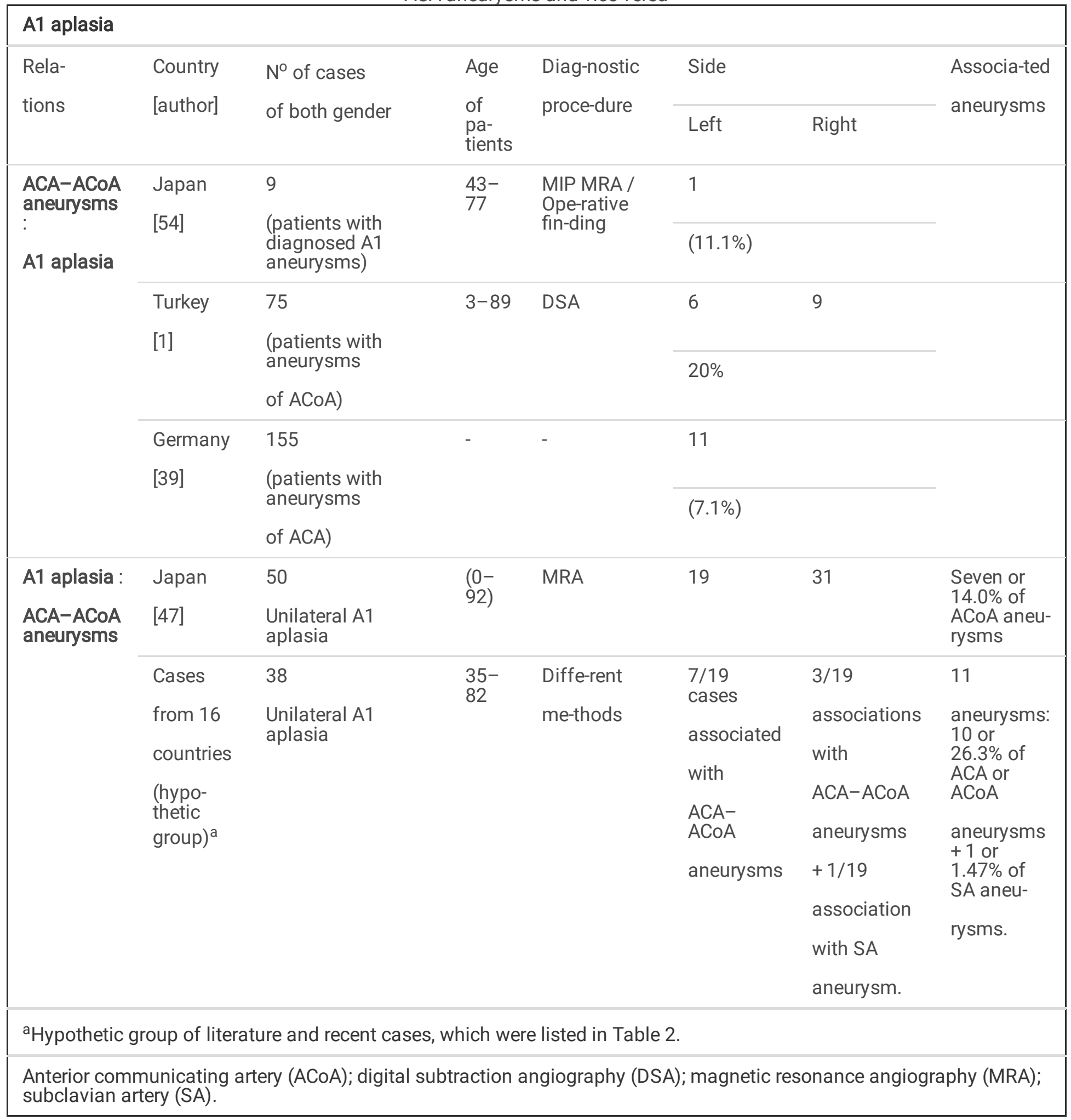

\section{Discussion}

The development of the circulatory system, as cited by Menshawi et al [32], begins with the formation of the six pairs of the primitive aortic arches within the $1.3 \mathrm{~mm}$ embryonic stage. These primitive arches stem from the aortic sac and course through the branchial arches, and then terminate in the ipsilateral dorsal aorta. The second and third aortic arches and a cranial continuation of the dorsal aorta are noticed in a $3.7 \mathrm{~mm}$ embryo [33]. The cervical part of the internal carotid artery 
(ICA) is derived primarily from the third aortic arch, whereas the other ICA segments represent cranial extensions of the dorsal aorta. In the embryos of 4-5.7 mm (28-30 days), the paired ICAs give the primitive maxillary artery and bifurcate into the cranial and caudal divisions. The cranial division is the precursor of the ACA, the anterior choroidal (AChA) and the middle cerebral (MCA) arteries, whereas the caudal division is the base of the posterior communicating (PCoA) and the posterior cerebral (PCA) arteries.

There are also other hypotheses about the ACA development. As cited by Niederberger et al [36], the ACA development initially refers to the three vessels and the presence of the anterior communicating plexus between them. The third $A 2$ or the median artery of the corpus callosum and the anterior communicating plexus usually regress. As presented by Moffat [33], in the human embryo of 4-5.7 mm, the cranial division of the primitive ICA constitutes the primitive olfactory artery (PO that terminates in the nasal fossa, while the secondary artery constitutes the medial olfactory artery; this latter artery will become the ACA, while the terminal portion of the POlA usually regresses. The aplasia of one or both ACAs is probably a consequence of the incorporation of the cranial branch of the primitive ICA into the PO $A$, the MCA and the AChA; however, the PO $\mathrm{PA}$ regresses and does not become the ACA from an unknown reason; the possible consequences are either that the normal ACA becomes bihemispheric in the case of unilateral ACA aplasia, or that MCA collaterals develop and supply the ACA territory in the case of bilateral ACA aplasia.

We found one case $(1 / 388)$ of unilateral aplasia, and one case $(1 / 388)$ of bilateral aplasia of the ACA in the presence of the ICA in the specimens of Serbian population. We did not include any case of ACA absence caused by the aplasia of the ICA. The frequency of less than one per cent was recorded also both in patients without vascular pathology of Turkish population [1], and in cadavers of Indian population [16]. In addition to that, Dimmick and Faulder [11] noted that A1 aplasia could be seen in 1-2\% of cases, which is supported in report by Siddiqi et al [46]. However, Kane et al [22] found 9.6\% of A1 aplasia in the angiograms of the patients investigated because of different pathological disorders excluding arteriovenous malformation or intracranial hemorrhage, whereas Alawad et al [2] found it in $4.2 \%$ of the patients with different pathology excluding cerebrovascular accidents.

Ağayev et al [1] noted that the genetic features of certain populations may affect the incidence of A1 aplasia; however, the studies in some countries did not confirm this finding. In Serbia, Ješić et al [19] discovered A1 aplasia in 2.9\% of the angiograms of 1000 patients with different pathology; however we found $0.51 \%$ of (unilateral and bilateral) ACA aplasia in cadavers autopsied due to different reasons and pathology as well. In Japan, Wanibuchi et al [54] discovered $11.1 \%$ of A1 aplasia among nine patients with the ACA aneurysms, whereas Uchino et al [47] recorded 50/891 (5.6\%) the patients with A1 aplasia suspected to suffer from cerebrovascular disease, while Nagahama et al [34] recorded 3.6\% of A1 aplasia among the 55 patients hospitalized because of the instrumentation of the posterior cervical spine. In addition, Uchino et al [47] found a higher frequency of aplasia of the right ACA (31) vs. the left ACA (19) aplasia in Japanese population.

We found one case of a bilateral ACA aplasia among 388 cases, while Fisher [14] found two cases among 414 cadavers. Cho [7] described it as a case report.

We think that previously cited frequencies of unilateral ACA aplasia should not be taken as fixed values, especially because some authors do not distinguish ACA hypoplasia from aplasia [6, 8, 17, 31, 43, 54], or aplasia from agenesis [8, 16, 17, 23, 42]. Wanibuchi et al [54] described A1 hypoplasia in the text, while they marked the same case as "aplasia" in the table. Han et al [17] marked aplasia in the legend of the Fig. 2, although (hypoplastic) A1 is visible on the right side in the first image and on the left side in their second image. Furthermore, Chuang et al [8] and Saikia et al [43] recognized hypoplastic A1 if its diameter is either $<1 \mathrm{~mm}$ or absent (invisible). Some clinicians pointed out that an acquired occlusion of the A1 segment in elderly patients may be diagnosed as A1 aplasia in the MRA [47], as well as that the presence of the Doppler signal could not exclude the possibility of A1 aplasia in the MRA [28]. According to the fact that there is a difficulty in distinguishing aplasia from hypoplasia of the A1 using the MRA or the DSA [8, 17, 36, 43, 54], we strongly believe that the anatomical, forensic or surgical dissections are the only relevant methods to use for their proper distinction and differentiation. In addition, from the anatomical point of view, the hypoplastic ACA is a morphologically formed vessel having its origin, 
course, distribution of side branches and termination, in contrast to the aplastic vessel. Further, the vascular source, i.e. the ICA should exist in the case of ACA aplasia, while it should be missing in the case of "ACA agenesis". Another example of a contradictory description could be seen in detecting the presence of "the ACoA" in the cases of ACA aplasia $[1,3,12,47]$. We think that in the case of unilateral (or bilateral) ACA aplasia, the ACoA cannot exist, because it develops from the plexiforme anastomosis of both ACAs [32, 33, 51]. According to the cistern of the lamina terminalis, the ACA course was divided into the pre- and postlaminar part of the ACA. A unique feature of the ACA is that it can be bihemispheric as in the cases of its hyperplasia, hypoplasia, or aplasia of the opposite ACA; however, we think that the ACoA is formed only in the first and second, but not in the third case.

As for the descriptions of the origin of the left and right ACAs from one ICA, as described by Burbank and Morris [5], and Kawaji et al [23], a persistent PO $A$ A probably assumed the role of the "second" ACA [32, 33, 52]. Similar descriptions were also found in the articles of Pokhrel and Bhatnagar [40], and Saikia et al [43], but their schema and/or images do not correspond to the text, i.e. these images presented bihemispheric ACAs.

Hendrikse et al [18] proved that the flow volume in the contralateral ICA was significantly increased in the subjects with A1 aplasia comparing to the subjects with both A1 parts and comparing to the volume flow in the ipsilateral ICA. Raghothaman and Pandit [41] suggested that the high flow in one ACA, as a consequence of the aplasia of the opposite ACA, could have led to the formation of a large aneurysm. Although we have not found it, we are also of the opinion that unilateral A1 aplasia predisposes an aneurysm formation, as Ağayev et al [1] and Lazzaro et al [29] reported. From the Tables 2 and 3 it can be seen that the aneurysm of the ACA could be expected in "each fourth case" of aplasia of the opposite ACA. Although Chuang et al [8] claimed that in the absence of the ICA occlusion, A1 hypoplasia/agenesis could be an independent contributor to the risk of ischemic stroke, we found that only 4/42 of all the analyzed literature cases of A1 aplasia were accompanied with cerebral infarctions $[4,5,12,25]$, contrary to the finding of $1 / 50$ A1 aplasia in the study by Uchino et al [47]. The data of the analyzed single cases from 16 countries in the Table 2 indicate that aneurysms of the right ACA were more frequent among the cases of the left ACA aplasia (6/20), than vice versa (3/18).

The two recent cases belong to a female and male cadaver, as Gunal et al [16] reported. In some retrospective studies the authors did not keep the evidence about the gender of patients with unilateral A1 aplasia [27], except for in those of Kwon's and Lee's [28], Uchino et al [47], and Ješić et al [19], which showed that it is more frequent among males.

We noticed vascular variations associated with ACA aplasia in the Table 2 as certain authors described. However, some of them were of unique kind, such as the unilateral PCoA aplasia [30, 35, 48], or the VA aplasia [4], or the persistence of primitive carotid-basilar anastomoses — the trigeminal artery [45], or the hypoglossal artery [15], or the proatlantal intersegmental artery [4], or an unusual course of the ACA in the A1 part [23,36, 42]. Another rather interesting fact is that associated vascular variations were not evidenced in the 16/38 literature cases of unilateral ACA aplasia.

Fisher [14] presented the two cases of bilateral partial aplasia of the ACA by schema, while Cho [7] discovered the total bilateral aplasia of the ACA and consequently developed a leptomeningeal collateral flow from the MCA in a 64-year-old man. We did not only find bilateral ACA aplasia associated with BA ectasia and the left PCoA hypoplasia, but also MCA collaterals developed in a 68-year-old male.

Although the two recent cases of unilateral and bilateral A1 aplasia were without cerebral aneurysm or infarction, we cannot conclude that A1 aplasia is a single entity. The reason is a relatively great frequency of cerebral aneurysms calculated according to the number of every one of the recent and literature cases of A1 aplasia, or the incidences of A1 aplasia in the specimens with ACA aneurysms presented in the previous tables. This is another reason why we should investigate and compare the relations of hypoplastic ACAs and the associated abnormalities with the presented findings in this article and available literature.

\section{Conclusion}

Page 17/23 
Both instances of unilateral and bilateral ACA aplasia were noted in $0.25 \%$ of cases of 388 specimens of Serbian population. Except for unilateral ACA aplasia, the case of bilateral aplasia is a true rarity. Both of the presented cases (of male and female gender) were not accompanied with cerebral pathology. The lack of distinction between ACA aplasia and hypoplasia found in the literature can be considered confusing and could prevent one from comparing these cases more thoroughly. Summarizing the recent and available literature cases of unilateral ACA aplasia, we discovered that it is a more frequent on the right side and among male specimens. In the end, relying on the evidence of a quarter of the studied cases, we would like to warn about the possibility of the occurrence of aneurysm of the opposite ACA.

\section{Declarations}

The authors have no relevant financial or non-financial interests to disclose.

\section{Funding}

This study was funded by the Ministry of Science and Technological Development of Republic of Serbia (Grant Nos: 451-039/2021-14/200113), Internal project of Faculty of Medicine, University of Niš (No. 38/20) and Slovenian Research Agency (P3-0293 research program).

\section{Author contribution:}

Milena Trandafilović: conceptualization, data collection, manuscript writing and editing

Ljiljana Vasović: conceptualization, manuscript editing

Slobodan Vlajković: data analysis, manuscript writing

Borisav Stojanović: data collection, data analysis

Marija Mladenović-Todorović: data collection, data analysis

Martina Drevenšek: conceptualization, manuscript writing and editing

\section{Acknowledgment}

This study was funded by the Ministry of Science and Technological Development of Republic of Serbia (Grant Nos: 451-039/2021-14/200113; 41018, 175092), Internal project of Faculty of Medicine, University of Niš (no. 38/20) and Slovenian Research Agency (P3-0293 research program).

\section{References}

1. Ağayev K, Önal B, Yavuz K, Ziyal MI (2005) The association of A1 segment hypoplasia/aplasia with anterior communicating artery aneurysms: A radiological study. Turkish Neurosurg 15:196-199. http://turkishneurosurgery.org.tr/abstract.php?id=60

2. Alawad AHM, Hussein MA, Hassan MA (2009) Morphology and normal variations of the cerebral arterial circle "of Willis" in Khartoum diagnostic centre. Khartoum Med J 2:215-219.

3. Babu AN, Babu LA, Raden M, Ahuja K (2006) Transient paraparesis due to right carotid stenosis with left anterior cerebral artery aplasia. Neurology 67:907. https://doi.org/10.1212/01.wnl.0000221810.42503.d6 
4. Buljan K, Hegeduš I, Gilman Kuric T, Salha T, Tomić S, Butković Soldo S, Buljan V, Šošić Đ (2015) Type I persistent proatlantal artery associated with fusiform subclavian artery aneurysm. Report of one case. Rev Med Chile 143:10811084. https://doi.org/10.4067/S0034-98872015000800018

5. Burbank NS, Morris PP (2005) Unique anomalous origin of the left anterior cerebral artery. AJNR Am J Neuroradiol 26:2533-2535. http://www.ajnr.org/content/26/10/2533.long

6. Chen H-W, Yen P-S, Lee C-C, Chang, PY, Lee S, Lee W, Ling C, Chou S (2004) Magnetic resonance angiographic evaluation of circle of Willis in general population: a morphologic study in 507 cases. Chin J Radiol 29:223229. https://www.semanticscholar.org/paper/Magnetic-Resonance-Angiographic-Evaluation-of-of-in-ChenYen/ecade90caad4caa054c542ca2438282fd98d1789

7. Cho YN (2001) Bilateral anterior cerebral artery aplasia. J Korean Neurol Assoc 29:276. https://www.jkna.org/journal/view.php?number=5914

8. Chuang Y-M, Liu C-Y, Pan P-Y, Lin C-P (2007) Anterior cerebral artery A1 segment hypoplasia may contribute to A1 hypoplasia syndrome. Eur Neurol 57:208-211. https://doi.org/10.1159/000099160

9. Dauber W (2007) Pocket atlas of human anatomy. Thieme, Stuttgart-New York.

10. Dawson AG, Wilson A, Maskova J, Murray AD, Reid JM, Kuhan G (2012) Hypoplastic internal carotid artery stenosis with low-lying carotid bifurcation causing cerebral ischemia. J Vasc Surg 56:14161418. https://doi.org/10.1016/j.jvs.2012.05.068

11. Dimmick SJ, Faulder KC (2009) Normal variants of the cerebral circulation at multidetector CT angiography. RadioGraphics 29:1027-1043. https://doi.org/10.1148/rg.294085730

12. Emsley HCA, Young CA, White RP (2006) Circle of Willis variation in a complex stroke presentation: a case report. BMC Neurology 6:13. https://doi.org/10.1186/1471-2377-6-13

13. Feldman BA (2013) Embryology and variations of cerebral arteries - a pictorial review. https://dx.doi.org/10.1594/ecr2013/C-2520

14. Fisher CM (1965) The circle of Willis: anatomical variations. Vasc Dis 2:99-105.

15. Gerlach J, Jensen HP, Spuler H, Viehweger G (1963) Traumatic carotico-cavernous fistula combined with persisting primitive hypoglossal artery. J Neurosurg 20:885-887. https://doi.org/10.3171/jns.1963.20.10.0885

16. Gunnal SA, Wabale RN, Farooqui MS (2013) Variations of anterior cerebral artery in human cadavers. Neurology Asia 18:249-259. http://neurology-asia.org/articles/neuroasia-2013-18(3)-249.pdf

17. Han YK, Kim S, Yoon CS, Lee YM, Kang HC, Lee JS, Kim Kim HD (2011) A1 segment hypoplasia/aplasia detected by magnetic resonance angiography in neuropediatric patients. J Korean Child Neurol Soc 19:231239. https://www.annchildneurol.org/journal/view.php?number=568

18. Hendrikse J, van Raamt F, van der Graaf J, Mali WPTM, van der Grond J (2005) Distribution of cerebral blood flow in the circle of Willis. Radiology 235:184-189. https://doi.org/10.1148/radiol.2351031799

19. Ješić A, Torbica S, Marić S, Popović S, Kozić D (2011) Anatomic variations of the anterior portion of the circle of Willis: an MR angiography study. Curr Top Neurol Psychiatr Relat Discip XIX:9-16.

20. Kachhara R, Nair S, Gupta AK (1998) Fenestration of the proximal anterior cerebral artery (A1) with aneurysm manifesting as subarachnoid hemorrhage. A case report. Neurol Med Chir (Tokyo) 38:409-

412. https://doi.org/10.2176/nmc.38.409

21. Kamath S (1981) Observations on the length and diameter of vessels forming the circle of Willis. J Anat 133:419423. https://www.ncbi.nlm.nih.gov/pmc/articles/PMC1167613/

22. Kane AG, Dillon WP, Barkovich AJ, Norman D, Dowd CF, Kane TT (1996) Reduced caliber of the internal carotid artery: a normal finding with ipsilateral absence or hypoplasia of the A1 segment. AJNR Am J Neuroradiol 17:12951301. https://www.ncbi.nlm.nih.gov/pmc/articles/PMC8338544/

Page 19/23 
23. Kawaji H, Amano S, Hiramatsu H, Sakai N, Kamio Y, Namba H (2014) Dissecting aneurysm at the proximal segment of the anterior cerebral artery associated with infraoptic course anterior cerebral artery. NMC Case Report J 1:1215. https://doi.org/10.2176/nmccrj.2013-0351

24. Klötzsch C, Bozzato A, Lammers G, Mull M, Lennartz B, Noth J (1999) Three-dimensional transcranial color-coded sonography of cerebral aneurysms. Stroke 30:2285-2290. https://doi.org/10.1161/01.STR.30.11.2285

25. Kothari MA, Pathare AV, Burkule NJ, Joshi VV, Chikhalikar AA, Dalvi SG (1990) Anterior cerebral artery territory infarction (a case report). J Postgrad Med [serial online] 36:230. https://pubmed.ncbi.nlm.nih.gov/2132252/

26. Krishnamoorthy T, Gupta AK, Bhattacharya RN, Rajesh BJ, Purkayastha S (2007) Anomalous origin of the callosomarginal artery from the A1 segment with an associated saccular aneurysm. AJNR Am J Neuroradiol 27:20752077. https://pubmed.ncbi.nlm.nih.gov/17110670/

27. Krzyżevski RM, Tomaszewski KA, Kochana M, Kopeć M, Klimek-Piotrowska W, Walocha JA (2015) Anatomical variations of the anterior communicating artery complex: gender relationship. Surg Radiol Anat 37:8186. https://doi.org/10.1007/s00276-014-1313-7

28. Kwon H-M, Lee Y-S (2005) Transcranial Doppler sonography evaluation of anterior cerebral artery hypoplasia or aplasia. J Neurol Sci 231:67-70. https://doi.org/10.1016/j.jns.2005.01.004

29. Lazzaro MA, Ouyang B, Chen M (2012) The role of circle of Willis anomalies in cerebral aneurysm rupture. J Neurointerv Surg 4:22-26. http://dx.doi.org/10.1136/jnis.2010.004358

30. Lee JH, Choi CG, Kim DK, Kim GE, Lee HK, Suh DC (2004) Relationship between circle of Willis morphology on 3D timeof-flight MR angiograms and transient ischemia during vascular clamping of the internal carotid artery during carotid endarterectomy. AJNR Am J Neuroradiol 25:558-564. https://pubmed.ncbi.nlm.nih.gov/15090341/

31. Malamateniou C, Adams ME, Srinivasan L, Allsop JM, Counsell SJ, Cowan FM, Hajnal JV, Rutherford MA (2009) The anatomic variations of the circle of Willis in preterm-at-term and term-born infants: an MR angiography study at 3T. AJNR Am J Neuroradiol 30:1955-1962. https://doi.org/10.3174/ajnr.A1724

32. Menshawi K, Mohr JP, Gutierrez J (2015) A functional perspective on the embryology and anatomy of the cerebral blood supply. J Stroke 17:144-158. https://doi.org/10.5853/jos.2015.17.2.144

33. Moffat DB (1962) The embryology of the arteries of the brain. Ann R Coll Surg Engl 30:368382. https://pubmed.ncbi.nlm.nih.gov/14475028/

34. Nagahama K, Sudo H, Abumi K, Ito M, Takahata M, Hiratsuka S, Kuroki K, Iwasaki N (2014) Anomalous vertebral and posterior communicating arteries as a risk factor in instrumentation of the posterior cervical spine. Bone Joint $\mathrm{J} 96-$ B:535-540. https://doi.org/10.1302/0301-620X.96B4.33210

35. Nayak SB (2008) Anomalous arteries at the base of the brain - a case report. Neuroanatomy 7:4548. https://www.semanticscholar.org/paper/Anomalous-arteries-at-the-base-of-the-brain-a-caseNayak/098a964527f36498d01bebd72c0ee032d04f9990

36. Niederberger E, Gauvrit JY, Morandi X, Carsin-Nicol B, Gauthier T, Ferré JC (2010) Anatomic variants of the anterior part of the cerebral arterial circle at multidetector computed tomography arteriography. J Neuroradiol 37:139147. https://doi.org/10.1016/j.neurad.2009.12.004

37. Patil PV, Patil AM, Apte AV, Attarde VY (2014) Anomalous origin of the left vertebral artery from carotid bulb seen as "trifurcation" of left common carotid artery with acute infarct in ipsilateral thalamus: a case report. J Neuroimaging 25:662-664. https://doi.org/10.1111/jon.12172

38. Pekcevik Y (2014) How to recognize the variations of the cerebral vasculature? CT angiography snapshot. https://dx.doi.org/10.1594/ecr2014/C-0412

39. Pia HW (1979) Aneurysms of the anterior cerebral artery. In: Pia HW, Langmaid C, Zierski J (eds) Cerebral aneurysms. Advances in diagnosis and therapy. Springer-Verlag, Berlin-Heidelberg, pp 109-110. 
40. Pokhrel R, Bhatnagar R (2013) A rare anomaly in circle of Willis. Med J Birendra Hosp 12:4142. https://doi.org/10.3126/mjsbh.v12i1.9093

41. Raghothaman A, Pandit L (2014) Large unruptured proximal (A1) anterior cerebral artery aneurysm with aplasia of the contralateral A1. Neurol India 62:80-82. https://doi.org/10.4103/0028-3886.128339

42. Rosenørn J, Ahlgren P, Ronde F (1985) Pre-optic origin of the anterior cerebral artery. Neuroradiology 27:275277. https://doi.org/10.1007/BF00344501

43. Saikia B, Handique A, Phukan P, Lynser D, Jamil M (2014) Study of anomalies in the circle of Willis using magnetic resonance angiography in north eastern India. J Anat Soc India 63:67-73. https://doi.org/10.1016/j.jasi.2014.05.007

44. Sakellaropoulos A, Mourgela S, Kyrlesi A, Warnke J-P (2008) Hypoplasia of multiple cerebral arteries: report of an unusual case. J Stroke Cerebrovasc Dis 17:161-163. https://doi.org/10.1016/j.jstrokecerebrovasdis.2008.01.011

45. Schwartz NE, Albers GW (2007) Acute strokes in the setting of a persistent primitive trigeminal artery. J Neurol Neurosurg Psychiatry 78:745. http://dx.doi.org/10.1136/bcr.2006.111773

46. Siddiqi H, Ansar T, Fasih S (2013) Variations in cerebral arterial circle of Willis in patients with hemorrhagic stroke: a computed tomography angiography study. J Ravalpindi Med Coll 17:215-218.

https://www.journalrmc.com/volumes/1395217935.pdf

47. Uchino A, Nomiyama K, Takase Y, Kudo S (2006) Anterior cerebral artery variations detected by MR angiography. Neuroradiology 48:647-652. https://doi.org/10.2176/nmc.38.409

48. Urbanski PP, Lenos A, Blum JC, Ziegler V, Griewing B, Schmitt R, Diegeler A, Dinkel M (2008) Does anatomical completeness of the circle of Willis correlate with sufficient cross-perfusion during unilateral cerebral perfusion? Eur $\mathrm{J}$ Cardiothorac Surg 33:402-408. https://doi.org/10.1016/j.ejcts.2007.12.021

49. Vasović L, Milenković Z, Pavlović S (2002) Comparative morphological variations and abnormalities of circles of Willis: a minireview including two personal cases. Neurosurg Rev 5:247-251. https://doi.org/10.1007/s10143-002-0227-8

50. Vasović LP (2006) Fetal azygos pericallosal artery. Clin Anat 19:327-331. https://doi.org/10.1002/ca.20189

51. Vasović L, Trandafilović M, Jovanović I, Ugrenović S, Vlajković S, Milić M, Đorđević G. (2013) Morphology of the cerebral arterial circle in the prenatal and postnatal period of Serbian population. Childs Nerv Syst 29:22492261. https://doi.org/10.1007/s00381-013-2151-3

52. Vasović L, Trandafilović M, Vlajković S, Jovanović I, Ugrenović S. Persistent primitive olfactory artery in Serbian population. Biomed Res Int 2013;2013:903460. https://doi.org/10.1155/2013/903460

53. Vasović L, Trandafilović M, Vlajković S, Jovanović I, Ugrenović S (2014) Anterior cerebral-anterior communicating complex in the postnatal period: from a fenestration to the multiplication of arteries. FU Med Biol 16:111. http://casopisi.junis.ni.ac.rs/index.php/FUMedBiol/article/view/240

54. Wanibuchi M, Kurokawa Y, Ishiguro M, Fujishige M, Inaba K (2001) Characteristics of aneurysms arising from the horizontal portion of the anterior cerebral artery. Surg Neurol 55:148-155. https://doi.org/10.1016/S00903019(01)00396-2

\section{Figures}




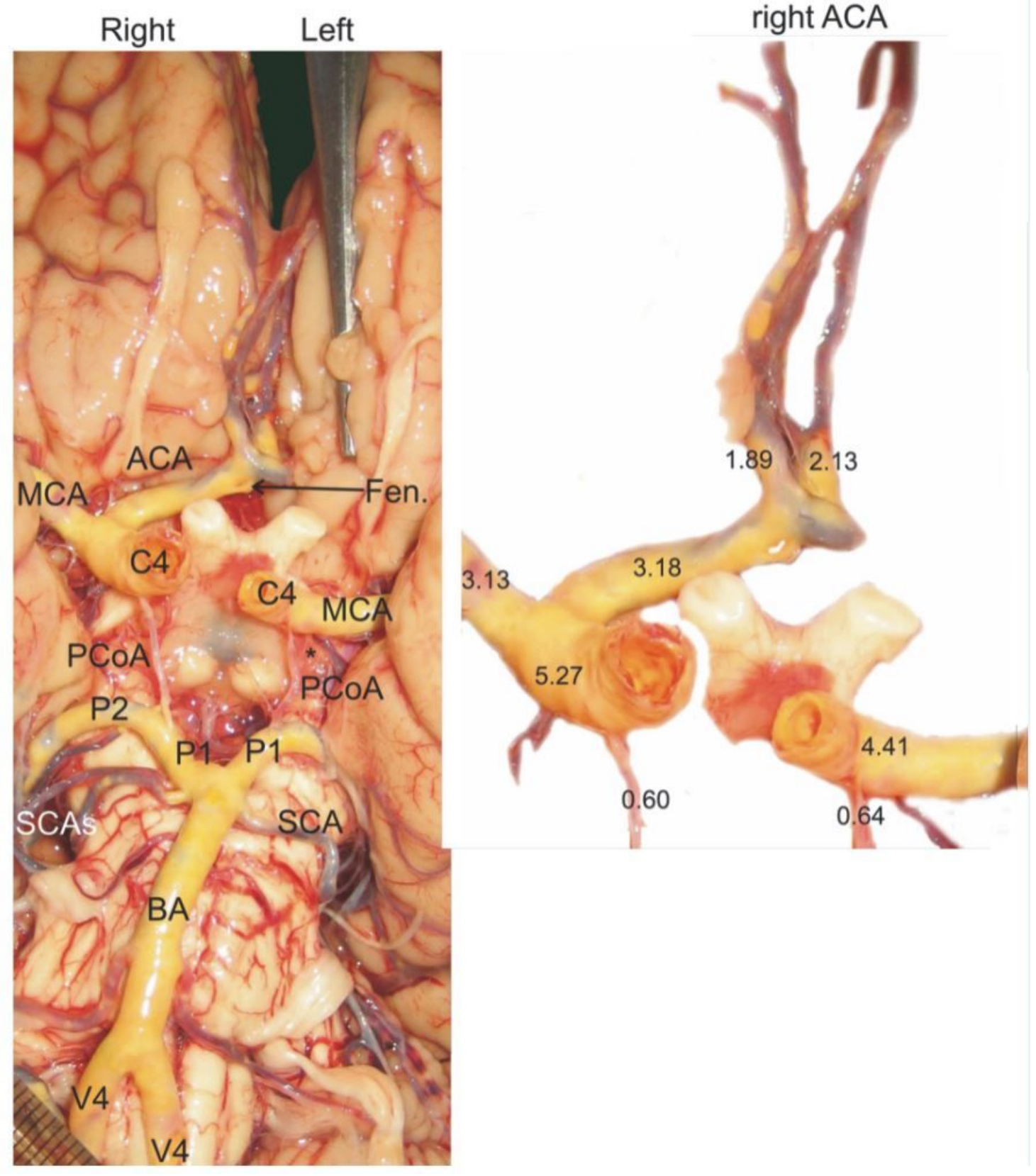

Figure 1

Case of unilateral aplasia of the anterior cerebral artery (ACA). View to some arteries of the carotid and vertebrobasilar systems on the brain base (left half of picture) and extracted arteries of the anterior segment of the cerebral arterial (semi)circle with the values of outer diameters (right half of picture). There are aplasia of the left ACA, small fenestration (fen) and bifurcation of the right ACA in the cistern of lamina terminalis, bilateral hypoplasia of the posterior communicating arteries (PCoAs), an initial partial duplication of the left PCoA, double right superior cerebellar arteries (SCAs), and atheromatous plaques in walls of cerebral vessels.

Cerebral part (C4) of the internal carotid artery; middle cerebral artery (MCA); pre-communicating part (P1) of the posterior cerebral artery; post-communicating part (P2) of the posterior cerebral artery; basilar artery (BA); intracranial part (V4) of the vertebral artery. 


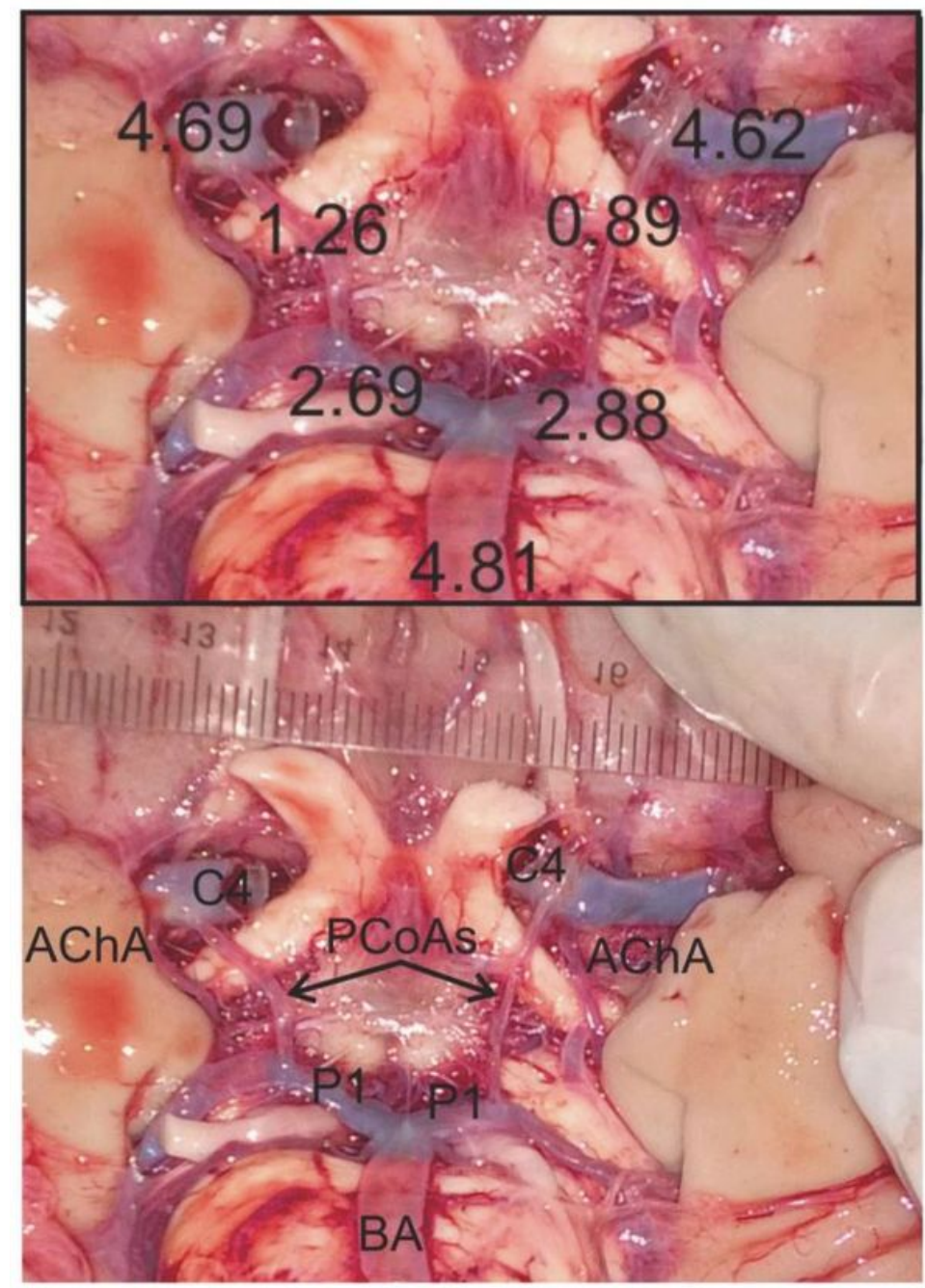

a)

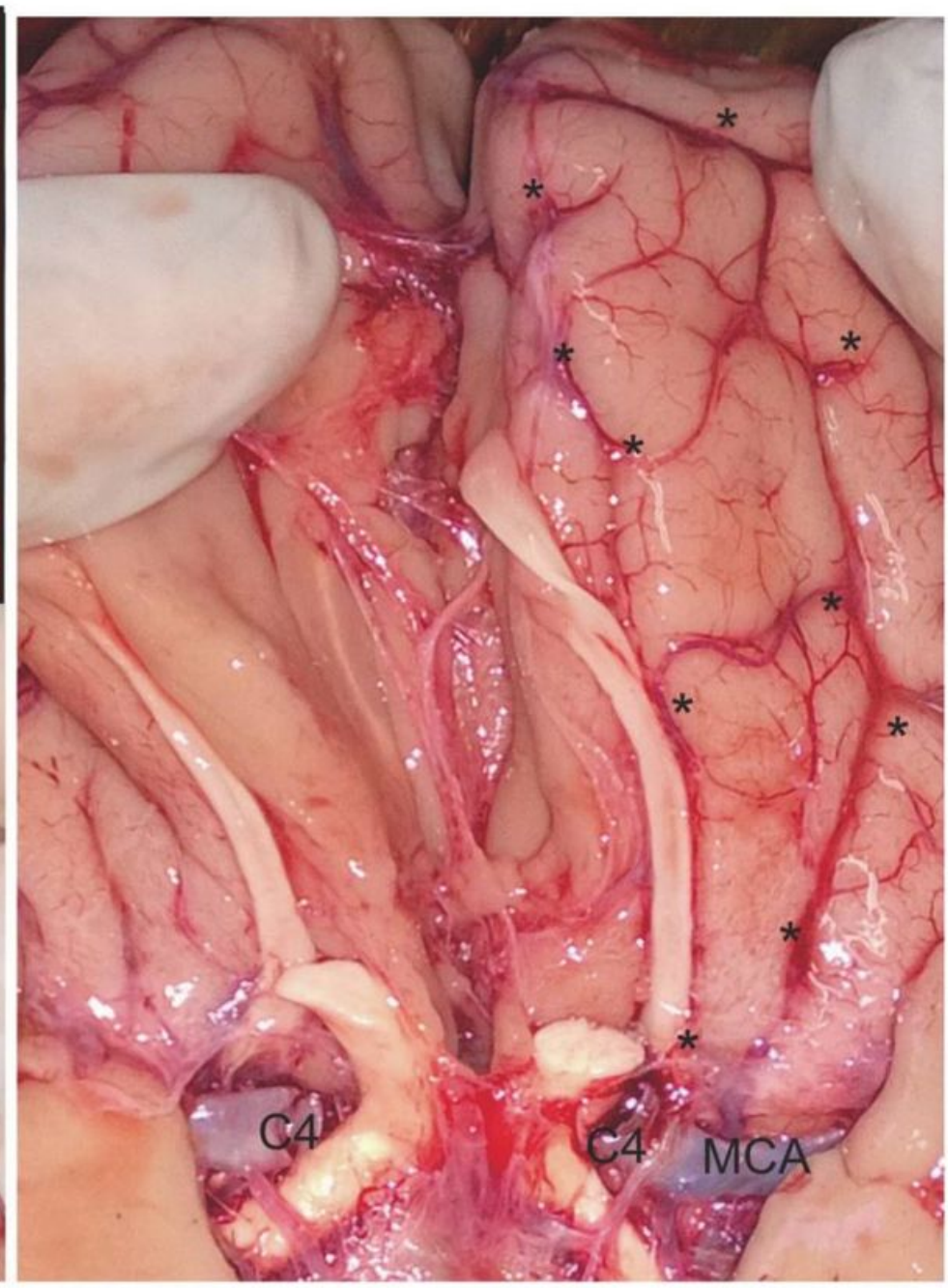

b)

Figure 2

Case of bilateral aplasia of the anterior cerebral artery (ACA). View to some arteries of the carotid and vertebrobasilar systems on the brain base (a) and inferomedial surface of the both frontal lobes (b). Some arteries of the cerebral arterial (semi)circle are marked (lower half), as well as the values of the outer diameters (upper half) in the insert "a". Collaterals* of the left middle cerebral artery are visible on the inferior surface of ipsilateral frontal lobe (b).

Cerebral part (C4) of the internal carotid artery; middle cerebral artery (MCA); posterior communicating arteries (PCoAs); anterior choroidal artery (AChA); pre-communicating part (P1) of the posterior cerebral artery; basilar artery (BA). 